\section{Absence of genome-wide changes in DNA methylation during development of the zebrafish}

Cps in the vertebrate genome are targets for DNA cytosine methyltransferases. Methylation leads to local transcriptional repression and is essential for normal development in the mouse ${ }^{1}$. Both gametogenesis and early embryoge- nesis of mouse are accompanied by changes in global levels of DNA methylation $^{2,3}$. Methylation levels are lowest in blastocyst DNA, but are restored to normal by the time of implantation. Several single-copy genes, repetitive sequences and transgenes have been shown to lose methylation in the early embryonic stages ${ }^{4}$. Notably, some imprinted regions have been shown to remain highly methylated through the blastocyst stage despite global hypomethylation ${ }^{4,5}$.

The functional significance of reduced methylation is not known, but it has been suggested that gametic methylation patterns must be reset in the early embryo to re-programme the genome for the next round of somatic development ${ }^{2,4}$. If so, it might be expected that global loss of $a$

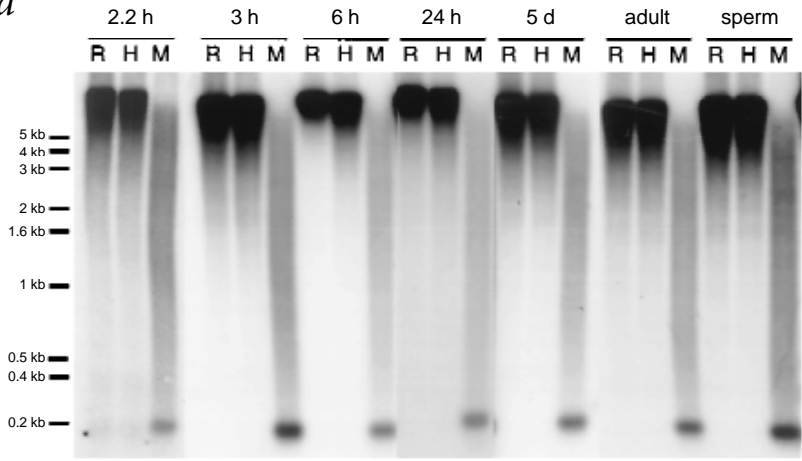

$\begin{array}{llllllllll}b & f g f-3 & 500 & 1000 & 1500 & 2000 & 2500 & 3000 & 3500 & 4000\end{array}$

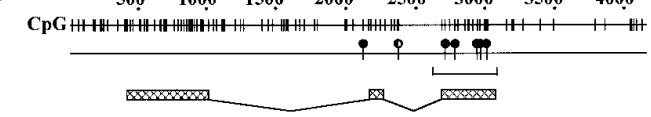

whn

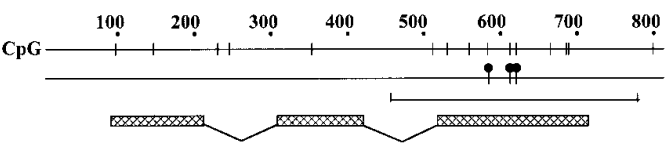

rag-I

$500 \quad 1000 \quad 1500 \quad 2000 \quad 2500 \quad 3000 \quad 3500 \quad 4000$

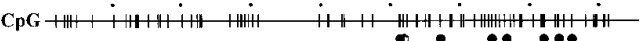
एक्ष

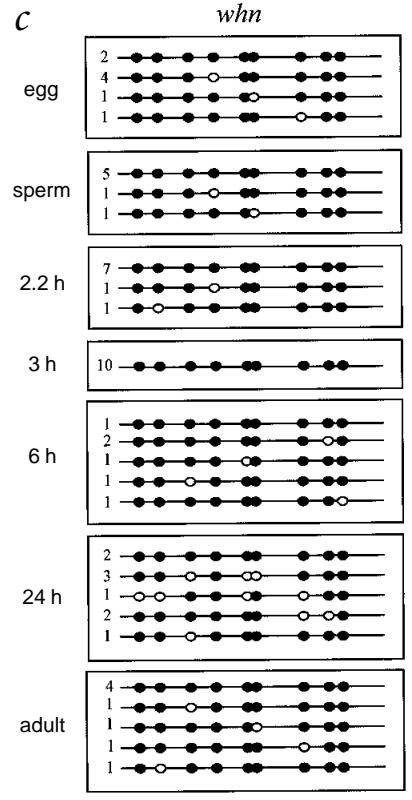

Fig. 1 High levels of DNA methylation in gametes and early embryonic stages of zebrafish. $\boldsymbol{a}$, A Southern blot of DNA samples ( $100 \mathrm{ng}$ ) from sperm, adult tail and embryonic stages ( $2.2 \mathrm{~h}-5 \mathrm{~d}$ ) hybridized with the DANA repeat probe. After digestion with EcoRI (R), EcoRI/Hpall (H) or EcoRI/Mspl (M), digests were assayed for completeness by co-incubating an aliquot (1/10) of the mixture with plasmid DNA (100 ng). Southern blots were prepared and hybridized using standard protocols. The DANA repeat probe was prepared by amplification of genomic DNA using primer sequences Dana-1, 5'-GGCGACRCAGTGGCGCAGTRGG-3', and Dana-2, 5'-TTTTCTTTTTGGCTTAGTCCC-3' (ref. 6). $\boldsymbol{b}$, Map-plots of CpG distribution (top line) and position of sites tested for methylation at fgf-3, whn and rag-1. Vertical lines indicate the position of CpG dinucleotides. Methylated sites in adult DNA, as determined from Southern blots, are indicated by filled circles; partially methylated sites by half-filled circles. The regions tested by genomic sequencing are bracketed. c. Bisulfite sequence analysis of all CpGs in above segments of fgf-3, whn and rag-1. The CpGs in each DNA sequence are represented by filled circles (methylated) and open circles (non-methylated). Methylation of rag1 in the egg sample was not analysed. The number of clones with each methylation pattern is shown on the left. Genomic sequence by bisulfite modification was obtained using standard procedures $5^{5,11}$. The amplified DNA was cloned into the pGEM-T Easy vector (Promega) and sequenced using a PE-Applied Biosystems 373A sequencer.
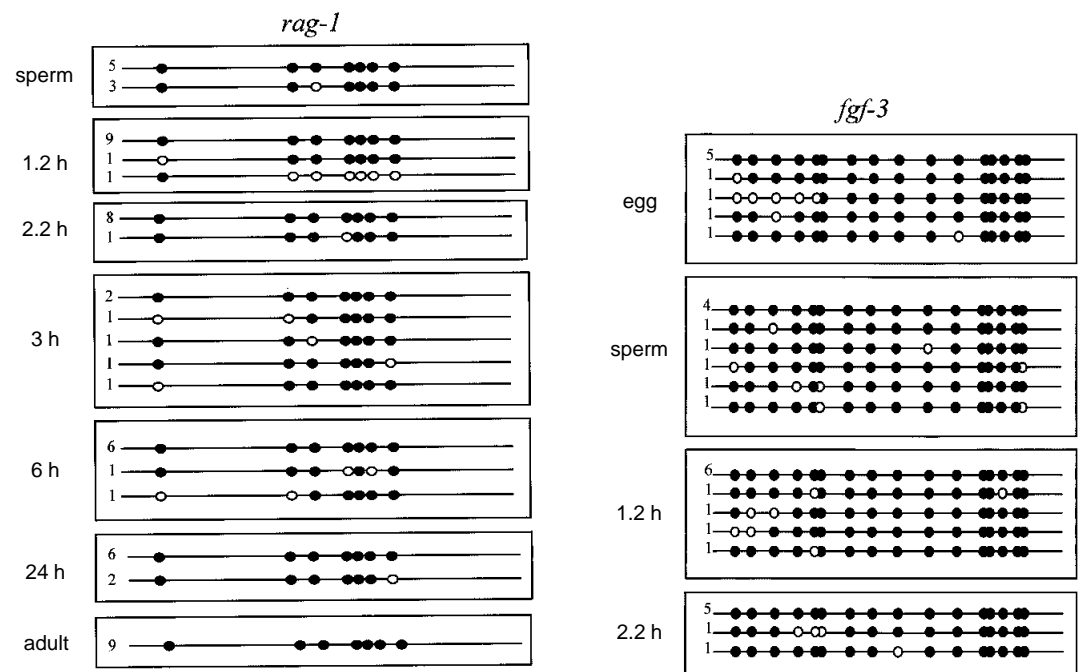

sperm

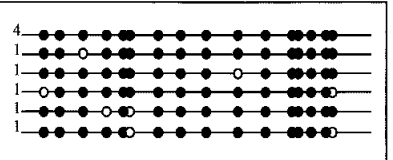

$1.2 \mathrm{~h}$

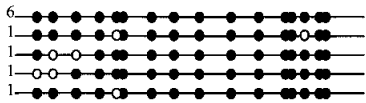

$2.2 \mathrm{~h}$

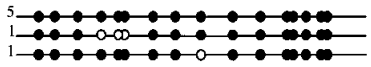

$3 \mathrm{~h}$

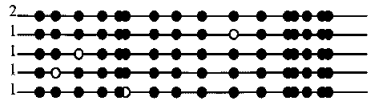

$6 \mathrm{~h}$
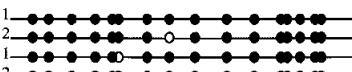

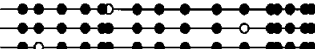

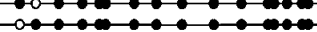

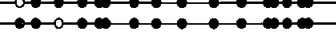

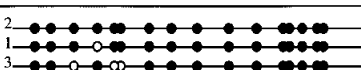

$\rightarrow-0-\infty, \cdots:-1:-\infty, \infty$

$1 \rightarrow-0-\infty-0,-\infty$

$2-0-1-0-1-0$

adult

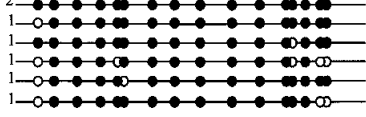


methylation would be a conserved feature of early vertebrate development. Because there is little data on other animal systems, we investigated methylation of repetitive DNA sequences and single-copy genes during development of the zebrafish. Embryos were collected at 2.2 hours (64-128 cells), 3 hours $(1,000-2,000$ cells), 6 hours (gastrula) and 24 hours (heartbeat) after fertilization. To analyse the methylation status of repeated sequences, we hybridized Southern blots of HpaII- and MspI-digested DNAs with a probe derived from the short interspersed repeat element (SINE), DANA, which makes up approximately $10 \%$ of the zebrafish genome 6 . We found that the DANA sequences in all samples were resistant to HpaII, but were cut by MspI to produce heterogeneous small fragments plus a prominent band at $180 \mathrm{bp}$ (Fig. 1a). Thus, contrary to a previous report ${ }^{7}$, we find that DANA repeats are consistently highly methylated at testable sites in sperm, adult and embryonic stages of the zebrafish. Equivalent results were obtained when blots were probed with ribosomal RNA genes (data not shown). The genome as a whole is highly methylated at HpaII sites in the developing embryo, sperm and adult, as indicated by ethidium bromide staining of the genomic DNA after electrophoresis (data not shown). We next tested three single-copy zebrafish genes chosen at random from the GenBank database: $w h n$ (related to the mouse gene Hfh11; ref. 8), fgf3 (a fibroblast growth factor gene ${ }^{9}$ ) and rag1 (associated with recombination ${ }^{10}$ ). Methylated regions in each gene were first determined by methylation-sensitive restriction endonucleases and Southern-blot hybridization of adult tail DNA (Fig. 1b). We then tested methylation of all CpGs in a short region in each of the three genes by bisulfite sequencing ${ }^{11}$ of DNA isolated from sperm, egg and embryonic stages, including at 1.2 hours (4-8 cell stage; Fig. 1c). Like repetitive sequences, these regions of single-copy genes are highly methylated in all samples (Fig. 2).

Our results indicate that development of a complex vertebrate need not involve global demethylation of the genome during embryogenesis. We did not detect passive loss of DNA methylation over several cell divisions, as seen in the mouse ${ }^{3}$. In theory, sudden global demethylation/de novo methylation may have escaped detection, but there is no reliable precedent for this. Why should the methylation status of zebrafish embryonic DNA be different from that of the mouse? There are several possible explanations. Two phenomena associated with DNA methylation in

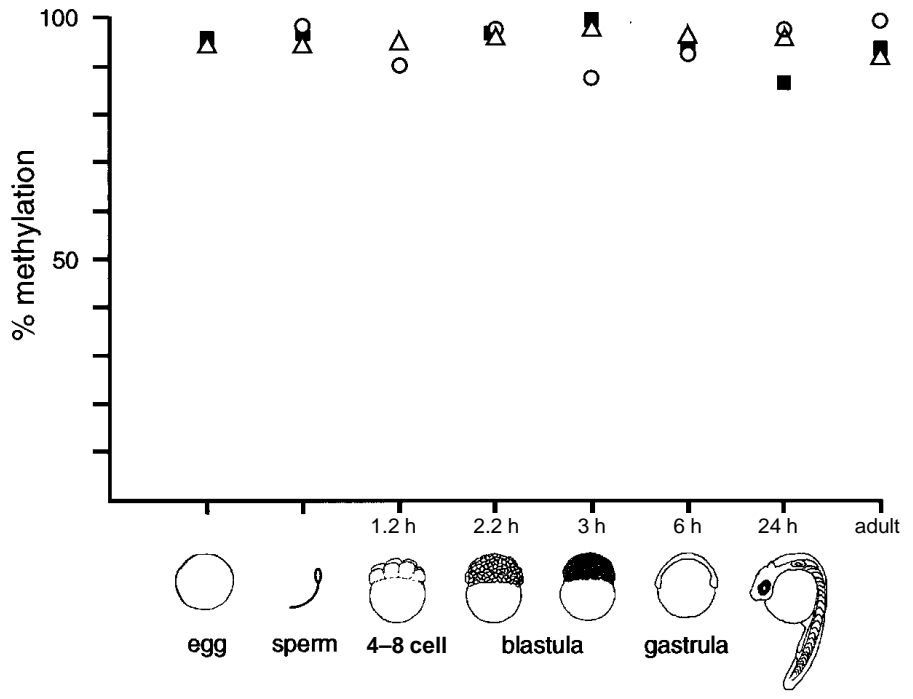

Fig. 2 Average methylation levels at three single-copy genes during early development of the zebrafish. Triangles, fgf3; filled squares, whn; circles, rag1.

mammals, parental imprinting and $\mathrm{X}$ inactivation, may not occur in zebrafish. No endogenous parentally imprinted genes have been described, and if imprinting were to exist, it is unlikely to affect essential genes, as androgenetic and gynogenetic zebrafish are viable ${ }^{12}$. In the mouse, however, androgenetic and gynogenetic embryos fail to develop to term $^{13,14}$. One hypothesis is that imprinting is a mammalian phenomenon and establishes the fetal-maternal relationship and development of the placenta ${ }^{15}$. There may be a relationship between embryonic loss of methylation and the requirement for an imprinted genome in the mouse. Parental alleles of some imprinted genes are differentially marked by methylation and a mechanism exists to maintain this mark in the preimplantation mouse embryo when global demethylation takes place $^{4,5,15}$. Low Dnmt activity at this time might prevent accidental de novo methylation of non-methylated (but perhaps silent) parental alleles without jeopardizing maintenance of the imprinting signal. Alternatively, loss of methylation from the mouse blastocyst genome may serve to erase methylation from the gene Xist, enabling random $\mathrm{X}$ inactivation to proceed in the post-implantation embryo ${ }^{15}$. In the absence of imprinting and $\mathrm{X}$ inactivation, the zebrafish would not need either of these mechanisms. A third possibility is raised by the observation that the mammalian genome (unlike that of other vertebrates) is activated very early during development ${ }^{16}$, suggesting that the loss of methylation from the mouse embryo may facilitate the early activation of zygotic transcription. Other vertebrates, such as the zebrafish, may not require input from the zygotic genome until later, and instead rely on maternal factors derived from the oocyte. In the meantime, the silent embryonic genome remains methylated.

\section{Acknowledgements}

We thank J. Lewis, S. Massey and P. Currie for staged zebrafish embryos; A. Greig and

J. Davidson for technical support; and S. Tweedie for critical reading of the manuscript. This work was supported by The Wellcome Trust and The Howard Hughes Medical Institute

\section{Donald Macleod, Victoria H. Clark} \& Adrian Bird Institute of Cell and Molecular Biology, University of Edinburgh, The King's Buildings, Edinburgh EH9 3JR, Scotland. Correspondence should be addressed to D.M. (e-mail: Donald.Macleod@ed.ac.uk).

1. Li, E., Bestor, T. \& Jaenisch, R. Cell 69, 915-926 (1992)

2. Monk, M., Boubelik, M. \& Lehnert, S. Development 99, 371-382 (1987)

3. Rougier, N. et al. Genes Dev. 12, 2108-2113 (1998)

4. Razin, A. \& Shemer, R. Hum. Mol. Genet. 4, 1751-1755 (1995).

5. Tremblay, K.D., Duran, K.L. \& Bartolomei, M.S. Mol. Cell. Biol. 17, 4322-4329 (1997).

6. Izsvák, Z., Ivics, Z., Garcia-Estefania, D., Fahrenkrug, S.C. \& Hackett, P.B. Proc. Natl Acad. Sci. USA 93 1077-1081 (1996).

7. Martin, C.C., Laforest, L., Akimenko M.-A. \& Ekker, M. Dev. Biol. 206, 189-205 (1999).

8. Shlake, T., Schorpp, M., Nehls, M. \& Boehm, T. Proc. Natl Acad. Sci. USA 94, 3842-3847 (1997).

9. Keifer, P., Strahle, U., Mason, I. \& Dickson, C. Oncogene 12, 1503-1511 (1996).

10. Willett, C.E., Cherry, J.J. \& Steiner, L.A. Immunogenetics 45, 394-404 (1997).

11. Frommer, M. et al. Proc. Natl Acad. Sci. USA 89, 1827-1831 (1992).

12. Corley-Smith, G.E., Lim, C.J. \& Brandhorst, B.P. Genetics 142, 1265-1276 (1996).

13. McGrath, J. \& Solter, D. Cell 37, 179-183 (1984).

14. Surani, M.A.H., Barton, S.C. \& Norris, M.L. Nature 308, 548-550 (1984).

15. Jaenisch, R. Trends in Genet. 13, 323-329 (1997)

16. Latham, K.E., Garrels, L.I., Chang, C. \& Solter, D. Development 112, 921-932 (1991) 